\section{Does Prolonged Infusion Allow Lower Daily Dose of Meropenem than Bolus Dosing?}

Antimicrobial dosage regimens based on pharmacodynamics can help to maximize antibacterial exposure, improve clinical outcomes, and reduce costs. Meropenem displays time-dependent killing, its efficacy depending on the period for which the concentration is above the minimum inhibitory concentration $(t>\mathrm{MIC})$, rather than peak concentrations. ${ }^{1,2}$ Meropenem has bacteriostatic activity at 20\%t> MIC and is bactericidal at $40 \% t>\mathrm{MIC}^{2-5}$ and dosing schedules have been selected on the basis of these characteristics. However, application of the known pharmacodynamics of meropenem could perhaps be used to alter the timing of delivery, in turn allowing the use of lower doses of meropenem with equivalent efficacy and net cost savings.

To date, studies assessing the potential benefit of prolonged intermittent infusions of meropenem have used Monte Carlo simulations. ${ }^{67}$ A Monte Carlo simulation is a statistical tool that attempts to mimic real-world situations by generating hypothetical trial data based on variation within the population for a number of characteristics. In the case of meropenem, a Monte Carlo simulation would use population data on clearance, volume of distribution, and MIC to generate thousands of hypothetical patients. Such a simulation could be used to measure the consistency of various dosage regimens in achieving surrogate markers of efficacy. To quantify consistency within the theoretical population, the portion of the population achieving the surrogate marker is calculated; this is termed the cumulative fraction of response. ${ }^{6,7}$

Lomaestro and Drusano ${ }^{6}$ performed a Monte Carlo simulation to assess the pharmacodynamics of meropenem using standard pharmacokinetic parameters and MIC values consistent with those of Pseudomonas aeruginosa. When the MIC was set at $1 \mathrm{mg} / \mathrm{L}$, the cumulative fraction of response for a 500-mg dose infused over $3 \mathrm{~h}$ q $8 \mathrm{~h}$ was similar to that of a 1 -g dose infused over $1 \mathrm{~h} \mathrm{q} 8 \mathrm{~h}(100 \%$ versus $99.28 \%) .{ }^{6}$ Kuti and others ${ }^{7}$ performed a similar Monte Carlo simulation using 30\%t> MIC and 50\% $t>$ MIC for bacteriostatic and bactericidal activity, respectively; they found that the cumulative fraction of response for meropenem $500 \mathrm{mg}$ infused over $3 \mathrm{~h} \mathrm{q} 8 \mathrm{~h}$ was similar to that of meropenem $1 \mathrm{~g}$ infused over $30 \mathrm{~min} \mathrm{q} 8 \mathrm{~h}$ for their P. aeruginosa isolates $(78.1 \%$ versus $82.0 \%$, respectively, for bacteriostatic activity; $73.5 \%$ versus $74.9 \%$, respectively, for bactericidal activity).

To date, there has been only one nonrandomized, open-label clinical trial examining the potential clinical effects of prolonged, intermittent infusion of meropenem. In that study, Itabashi compared the clinical efficacy of meropenem $500 \mathrm{mg}$ given twice daily over $4 \mathrm{~h}$ and over $1 \mathrm{~h}$ in 42 patients with severe pneumonia. Eighteen of the patients received the intervention (4-h infusion twice daily), and the remaining 24 received the 1 - $h$ infusion twice daily. The mortality rate was significantly lower for patients in the prolonged-infusion group than for those in the 1-h infusion group $(5.6 \%$ versus $37.5 \% ; p<0.05)$. Although pharmacokinetic data were not assessed and the sample size was small, these results suggest that prolonged infusion of meropenem could lead to better clinical outcomes. However, the distribution of infecting organisms between the 2 groups differed (with more Klebsiella pneumoniae and $P$. aeruginosa in the 1-h infusion group); as such, the difference in outcome might not have been solely based on the infusion time for meropenem. Canadian clinicians should also recognize that the total daily dose for the 2 treatment arms was similar, and this was less than the usual treatment dose used in Canada.

These studies have shown the potential for achieving equally effective surrogate and clinical outcomes with lower doses of meropenem given over a more prolonged infusion time. The logistic difficulties of prolonged infusion, including the potential for incompatibilities with simultaneously infused drugs or physiochemical stability of meropenem, must be considered if a prolonged infusion time is adopted. Adequately powered clinical studies comparing traditional doses and traditional administration times of meropenem with lower total daily doses given as a 3-h infusion, with evaluation of pharmacokinetic and pharmacodynamic surrogate markers and clinically important end points, would help to confirm noninferiority in clinical success rates and potential savings in drug costs. We believe that the available investigational data demonstrate the potential benefit of a prolonged intermittent infusion of a lower total daily dose of meropenem.

References

1. Ambrose PG, Bhavnani SM, Rubino CM, Louie A, Gumbo T, Forrest A, et al. Pharmacokinetics-pharmacodynamics of antimicrobial therapy: it's not just for mice anymore. Clin Infect Dis 2007;44(1):79-86. Erratum in: Clin Infect Dis 2007;44(4):624.

2. Nicolau DP. Pharmacokinetic and pharmacodynamic properties of meropenem. Clin Infect Dis 2008;47 Suppl 1:S32-S40.

3. Drusano GL. Prevention of resistance: a goal for dose selection for antimicrobial agents. Clin Infect Dis 2003;36(Suppl 1):S42-S50.

4. Santos Filho L, Eagye KJ, Kuti JL, Nicolau DP. Addressing resistance evolution in Pseudomonas aeruginosa using pharmacodynamic modelling: application to meropenem dosage and combination therapy. Clin Microbiol Infect 2007;13(6):579-585.

5. Craig WA, Ebert $S$, Watanabe Y. Differences in time above MIC required for efficacy of B-lactams in animal infection models [abstract 86]. In: Abstracts of the 33rd Interscience Conference on Antimicrobial Agents and Chemotherapy; San Francisco (CA). Washington (DC): American Society for Microbiology; 1993.

6. Lomaestro BM, Drusano GL. Pharmacodynamic evaluation of extending the administration time of meropenem using a Monte Carlo simulation. Antimicrob Agents Chemother 2005;49(1):461-463.

7. Kuti JL, Dandekar PK, Nightingale CH, Nicolau DP. Use of Monte Carlo simulation to design an optimized pharmacodynamic dosing strategy for meropenem. J Clin Pharmacol 2003;43(10):1116-1123. Erratum in: J Clin Pharmacol 2005;45(3):357.

8. Itabashi S. [Clinical efficacy of prolonged (4 hour) drip infusion of meropenem against severe pneumonia]. Jpn J Antibiot 2007;60(3): 161-170. Japanese.

Eric Poulin, BScPharm

St Paul's Hospital

Vancouver, British Columbia

Glen Brown, BSC(Pharm), PharmD, FCSHP, BCPS

Pharmacy

Providence Health Care

Vancouver, British Columbia 